MINI REVIEW

\title{
The role of octreoscan in thyroid eye disease
}

\author{
G E Krassas and G J Kahaly ${ }^{1}$ \\ Department of Endocrinology and Metabolism, Panagia Hospital, Thessaloniki, Greece and ${ }^{1}$ Department of Endocrinology and Metabolism, \\ Gutenberg University Hospital, Mainz, Germany \\ (Correspondence should be addressed to G E Krassas, Endocrine Department, Panagia General Hospital, N Plastira 22, N Krini, GR-54622, Thessaloniki, \\ Greece)
}

\begin{abstract}
Until recently there was no imaging technique available which could demonstrate pathological changes in orbital tissues and could be regarded as a reliable measure of inflammation in thyroid eye disease (TED). Pentetreotide (a synthetic derivative of somatostatin) labelled with ${ }^{111}$ In has been used to localize tumours which possess surface or membrane receptors for somatostatin in vivo using a gamma camera (1). This technique visualizes somatostatin receptors in endocrine-related tumours in vivo and predicts the inhibitory effect of the somatostatin analogue octreotide on hormone secretion by the tumours (1). By applying ${ }^{111}$ In-DTPA-D-Phe octreotide scintigraphy (octreoscan), accumulation of the radionuclide was also detected in both the thyroid and orbit of patients with Graves' disease (2-4). If peak activity in the orbit $5 \mathrm{~h}$ after injection of radiolabelled octreotide is set at $100 \%$, a decrease to $40 \pm 4 \%$ is found at $24 \mathrm{~h}$, significantly different from the decrease in blood pool radioactivity, which is $15 \pm 4 \%$ at $24 \mathrm{~h}$. Accumulation of the radionuclide is most probably due to the presence in the orbital tissue of activated lymphocytes bearing somatostatin receptors (5). Alternative explanations are binding to receptors on other cell types (e.g. myoblasts, fibroblasts or endothelial cells) or local blood pooling due to venous stasis by the autoimmune orbital inflammation.
\end{abstract}

European Journal of Endocrinology 140 373-375

Clinically active thyroid eye disease (TED) is characterized by mononuclear infiltration and inflammation of the orbital adipose/connective tissue which evolves into fibrosis (5-7). It is suggested that intervention using immunosuppressive drugs or retrobulbar irradiation will be most successful if applied in the phase of active inflammation, while rehabilitative surgery, i.e. orbital decompression and eye muscle and lid surgery, should be carried out in the stable end-phase. Therefore, to decide which treatment should be given it is important to determine the clinical phase of the disease in the individual patient. The clinical activity score (CAS), using a cut-off score of 3 or more of 7 points, is specific but sensitivity is not high (8). Imaging techniques (magnetic resonance imaging) and laboratory methods, e.g. glycosaminoglycan excretion in urine, have also been applied to evaluate clinical activity of the disease $(9,10)$. Results of octreoscan in patients with TED correlate well with CAS and the T2 relaxation time of the extra-ocular rectus muscles $(2,11)$. Orbital pentetreotide accumulation is significantly higher in subjects with active TED; the uptake in the inactive group is close to that in control subjects, in whom no specific uptake is observed (12).

These data demonstrate that a positive orbital octreoscan indicates clinically active eye disease in which immunosuppressive treatment might be of therapeutic benefit, in contrast to the fibrotic end-stage. Indeed, successful immunosuppression with prednisone, orbital irradiation, intravenous immunoglobulin or very recently with somatostatin analogues, has been found to be associated with a fall in orbital pentetreotide uptake $(2,12-18)$.

Thus, orbital octreoscan is mainly indicated in clinical practice to select patients with TED who will benefit from immunosuppression. To this end one must know the predictive value of orbital octreoscan for the outcome of immunosuppressive treatment. In one study (12) when a clinical improvement of TED was considered, as indicated by a decrease in the NOSPECS score (No symptoms, no signs, Only signs, Symptoms, Proptosis, Extraocular muscles involvement, Ceratitis, Sight loss) and in the CAS after 3 months of therapy with octreotide, the positive and negative predictive values of octreoscan were 87 and $100 \%$ respectively. When lanreotide was used, the positive predictive value was $90 \%$ (14). In a recent paper (16), 14 of 16 patients with TED, with an orbit to brain ratio higher than $10,4 \mathrm{~h}$ postinjection, responded to steroid and radiotherapy, in contrast to none of 4 patients with a ratio less than 10 . Regarding the evaluation of activity of TED, it was found that when an orbit to brain pentetreotide ratio greater than $10,4 \mathrm{~h}$ postinjection, was chosen as the cut-off point, a sensitivity of $94 \%$ and a specificity of $100 \%$ were given (13). 
Wide differences exist between various studies regarding the administered dose of radionuclide, the time interval after injection for determining the orbital uptake, the selection of orbital slices for quantification of the orbital uptake, and the method of correction for background radioactivity (19-21). The earlier studies used rather large doses of pentetreotide and preferred to measure orbital uptake $24 \mathrm{~h}$ postinjection $(2,12,14$, 21), while later studies administered a low dose and measured orbital uptake only $2 \mathrm{~h}$ postinjection arguing that the low dose decreases the radiation burden and the cost of the examination $(15,22)$. Nevertheless, a low dose might cause problems in count statistics and at $2 \mathrm{~h}$ postinjection, $12 \%$ of the dose is still in the blood pool causing high background uptake. Furthermore, the radiation burden received from a high dose of 222 $\mathrm{MBq}$ is $16 \mathrm{mSV}$, in the same order as that from chest computed tomography or angiography (22). The inference is that in predicting the response to subsequent immunosuppression a $4 \mathrm{~h}$ scan is preferred, when a low dose is chosen (23).

However, any remaining orbital radioactivity at $24 \mathrm{~h}$ after administering a high dose might represent a greater degree of specific tissue binding, possibly enhancing both the differential diagnostic and predictive value of octreoscan (19). Another technical problem is the selection of regions of interest, which may result in considerable intra- and interobserver variation. Single photon emission computed tomography (SPECT) images are obviously required and measuring uptake in a number of orbital slices from SPECT images is of great advantage in the quantification of the results (19). The left temporal skull area $(2,12,14)$ was originally used for correction for background activity. Recently it was suggested that at least part of this radioactivity is due to uptake in the parotic gland (19, 23) and the authors recommended background measurements in the occipital skull area. The brain itself is used to measure background $(13,15,21)$ but for most investigators it seems less suitable to correct for blood pool radioactivity.

In a recent paper, analysis of inter- and intraobserver variability and reproducibility in the evaluation of orbital SPECT images was performed (24). For the right and the left orbit, the interobserver variance proportion was 90 and 79\% respectively. Intraobserver reliability for both orbits was 88, 89, 97 and 98\% respectively for four different observers, and intraclass correlation as a measure of multiple observer reproducibility was $94 \%$. Thus, due to the increased interobserver variance proportion and the high variation in intraobserver reliability, evaluation of orbital octreoscan has to be done by the same and experienced observer, leading to representative and comparable data.

In conclusion, a positive orbital octreoscan in patients with TED indicates clinically active eye disease in which immunosuppressive treatment might be of therapeutic benefit. Due to high sensitivity this technique may be regarded as a semi-objective tool in evaluating patients with TED, both at initial stages as well as during treatment. However, the following limitations restrict the widespread use of this technique. First, it is an expensive method with a non-negligible radiation burden. Secondly, it is non-specific, i.e. positive octreoscans may be obtained in patients with orbital diseases such as meningioma, myositis, lymphoma, granulomatosis, sarcoidosis and Wegener's disease, as well as sinusitis and infections of the nasal mucosa. Last, orbital octreoscan does not permit detailed orbital imaging, i.e. evaluation of eye muscle swelling. Thus, it remains to be seen if orbital octreoscan will become a widely available tool in the management of patients with TED.

\section{References}

1 Lamberts SWJ, Bakker WH, Reubi JC \& Krenning EP. Somatostatin-receptor imaging in the localization of endocrine tumours. New England Journal of Medicine 1990323 1246-1249.

2 Postema PTE, Krenning EP, Wijngaarde R, Kooy PPM, Oei HY, van den Bosch WA et al. (111-In-DTPA-D-Phe1) octreotide scintigraphy in thyroidal and orbital Graves' disease: a parameter for disease activity? Journal of Clinical Endocrinology and Metabolism 199479 1845-1851.

3 Krenning EP, Oei HY, Kooij PPM, Wijngaarde R, Lamberts SMJ, Wiersinga WM et al. Thyroid and orbital radiolabeled somatostatin (SS) accumulation in Graves' disease and Graves' ophthalmopathy. Journal of Nuclear Medicine 199233894.

4 Krenning EP, Kwekkeboom DJ, Bakker WH, Breeman WAP, Kooiz PPM, Oei HY et al. Somatostatin receptor scintigraphy with (III-In-DTAP-D-Phe1) and (123-I-Tyr3)-octreotide: the Rotterdam experience with more than 1000 patients. European Journal of Nuclear Medicine 199320 716-731.

5 Kahaly G, Hansen C, Felke B \& Dienes HP. Immunohistochemical staining of retrobulbar adipose tissue in Graves' ophthalmopathy. Clinical Immunology and Immunopathology 199473 53-62.

6 Hales IB \& Rundle FF. Ocular changes in Graves' disease. A longterm follow-up study. Quarterly Journal of Medicine 196029 113126.

7 Perros P, Crombie Al \& Kendall-Taylor P. Natural history of thyroid associated ophthalmopathy. Clinical Endocrinology 1995 $4242-50$.

8 Mourits MP, Koornneef L, Wiersinga WM, Prummel MF, Berghout A \& Van der Gaag R. Clinical criteria for the assessment of disease activity in Graves' ophthalmopathy: a novel approach. British Journal of Ophthalmology 198973 639-644.

9 Hosten N, Sander B, Cordes M, Schubert CJ, Schorner W \& Felix R. Graves' ophthalmopathy: MR imaging of the orbits. Radiology $1989172759-762$.

10 Kahaly G, Schuler M, Sewell AC, Bernard G, Beyer J \& Krause U. Urinary glycosaminoglycans in Graves' ophthalmopathy. Clinical Endocrinology 199033 35-44.

11 Kahaly G, Diaz M, Just M, Beyer J \& Lieb N. Role of octreoscan and correlation with MR imaging in Graves' ophthalmopathy. Thyroid $19955107-111$

12 Krassas GE, Dumas A, Pontikides N \& Kaltsas Th. Somatostatin receptor scintigraphy and octreotide treatment in patients with thyroid eye disease. Clinical Endocrinology $199542571-$ 580 .

13 Kahaly G, Diaz M, Hahn K, Beyer J \& Bockisch A. Indium 111pentetreotide scintigraphy in Graves' ophthalmopathy. Journal of Nuclear Medicine 199536 550-554.

14 Krassas GE, Kaltsas Th, Dumas A, Pontikides N \& Tolis G. Lanreotide in the treatment of patients with thyroid eye disease. European Journal of Endocrinology 1997136 416-422. 
15 Moncayo R, Baldnisera I, Decristoforo C, Kendler D \& Donnemiller E. Evaluation of immunological mechanisms mediating thyroidassociated ophthalmopathy by radionuclide imaging using somatostatin analog 111-In-octreotide. Thyroid 19977 21-29.

16 Kahaly G, Görges R, Diaz M, Hommel G \& Bockisch A. Indium111-pentetreotide in Graves' Disease. Journal of Nuclear Medicine $199839533-536$.

17 Krassas GE, Dumas A, Kaltsas Th, Halkias A \& Pontikides N. Somatostatin receptor scintigraphy before and after treatment with somatostatin analogues in patients with thyroid eye disease. Thyroid 1999 9 47-52.

18 Kahaly GJ \& Förster GJ. Somatostatin receptor scintigraphy in thyroid eye disease. Thyroid 19988 549-552.

19 Wiersinga WM, Gerding MN, Prummel MF \& Krenning EP. Octreotide scintigraphy in thyroidal and orbital Graves' disease. Thyroid 19988 433-436.

20 Durak I, Durak H, Ergin M, Yurekli Y \& Kaynak S. Somatostatin receptors in the orbit. Clinical Nuclear Medicine $199520237-242$.
21 Bohuslavizki KH, Oberwohrmann S, Brenner W, Eberhardt JU, Monig H, Clausen $\mathrm{M}$ et al. 111-In-octreotide imaging in patients with longstanding Graves' ophthalmopathy. Nuclear Medicine Communications $199516912-916$.

22 Krassas GE, Dumas A \& Moncayo R. Octreoscan in Graves' ophthalmopathy (letters to the editor). Thyroid 19977 805-806.

23 Gerding MN, van der Zant FM, van Royen EA, Koornneef L, Krenning EP, Wiersinga WM \& Poummel MF. Octreotidescintigraphy is a disease-activity parameter in Graves' ophthalmopathy. Clinical Endocrinology 199950 373-379.

24 Förster GJ, Krummenauer F, Nickel O \& Kahaly GJ. Reproducibility and inter-/intra-observer variability of somatostatin-receptor scintigraphy in Graves' ophthalmopathy. Thyroid 1999 (In Press).

Received 20 January 1999

Accepted 12 February 1999 